LAWRENCE LIVERMORE NATIONAL LABORATORY
PLEIADES: A Picosecond Compton Scattering X-Ray Source for Advanced Backlighting and TimeResolved Material Studies

D.J. Gibson, S.G. Anderson, C.P.J. Barty, S.M. Betts, R. Booth, W.J. Brown, J.K. Crane, R.R. Cross, D.N. Fittinghoff, F.V. Hartemann, J. Kuba, G.P. Le Sage, D.R. Slaughter, A.M. Tremaine, A.J. Wooten, E.P. Hartouni, P.T. Springer, J.B. Rosenzweig

May, 2004

Physics of Plasmas 
This document was prepared as an account of work sponsored by an agency of the United States Government. Neither the United States Government nor the University of California nor any of their employees, makes any warranty, express or implied, or assumes any legal liability or responsibility for the accuracy, completeness, or usefulness of any information, apparatus, product, or process disclosed, or represents that its use would not infringe privately owned rights. Reference herein to any specific commercial product, process, or service by trade name, trademark, manufacturer, or otherwise, does not necessarily constitute or imply its endorsement, recommendation, or favoring by the United States Government or the University of California. The views and opinions of authors expressed herein do not necessarily state or reflect those of the United States Government or the University of California, and shall not be used for advertising or product endorsement purposes.

This work was performed under the auspices of the U.S. Department of Energy by University of California, Lawrence Livermore National Laboratory under Contract W-7405-Eng-48. 


\title{
PLEIADES: a picosecond Compton scattering $x$-ray source for advanced backlighting and time-resolved material studies
}

\author{
David J. Gibson', Scott G. Anderson, Christopher P. J. Barty, Shawn M. Betts, Rex Booth, \\ Winthrop J. Brown, John K. Crane, Robert R. Cross, David N. Fittinghoff, Fred V. Hartemann, \\ Jaroslav Kuba, Gregory P. Le Sage, Aaron M. Tremaine, and Paul T. Springer \\ Lawrence Livermore National Laboratory, L-280, P.O. Box 808 Livermore, CA, USA 94551- \\ 0808 \\ James B. Rosenzweig \\ University of California at Los Angeles, Department of Physics and Astronomy, Box 951547, \\ Los Angeles, CA 90095-1547
}

\begin{abstract}
The PLEIADES (Picosecond Laser-Electron Inter-Action for the Dynamical Evaluation of Structures) facility has produced first light at $70 \mathrm{keV}$. This milestone offers a new opportunity to develop laser-driven, compact, tunable x-ray sources for critical applications such as diagnostics for the National Ignition Facility and time-resolved material studies. The electron beam was focused to $50 \mu \mathrm{m} \mathrm{rms}$, at $57 \mathrm{MeV}$, with $260 \mathrm{pC}$ of charge, a relative energy spread of $0.2 \%$, and a normalized emittance of $5 \mathrm{~mm}$ mrad horizontally and $13 \mathrm{~mm}$ mrad vertically. The scattered 820-nm laser pulse had an energy of $180 \mathrm{~mJ}$ and a duration of $54 \mathrm{fs}$. Initial x-rays were captured with a cooled chargecoupled device using a Cesium Iodide scintillator; the peak photon energy was approximately 78

a gibson23@1lnl.gov
\end{abstract}


$\mathrm{keV}$, with a total $\mathrm{x}$-ray flux of $1.3 \times 10^{6}$ photons/shot, and the observed angular distribution found to agree very well with three-dimensional codes. Simple K-edge radiography of a tantalum foil showed good agreement with the theoretical divergence-angle dependence of the x-ray energy. Optimization of the x-ray dose is currently underway, with the goal of reaching $10^{8}$ photons per shot and a peak brightness approaching $10^{20}$ photons $/ \mathrm{mm}^{2} / \mathrm{mrad}^{2} / \mathrm{s} / 0.1 \%$ bandwidth.

Keywords: Hard x-ray source, Compton scattering, $4^{\text {th }}$ generation light sources, photon-electron beam interaction 


\section{INTRODUCTION}

The unique conditions of atomic-scale interactions have led to a recent experimental push to develop high-brightness, ultrashort hard x-ray sources. The energy levels relevant to the inner-shell electron properties, which are responsible for most fundamental atomic-scale effects, require photon energies well above those generated by modern ultrafast laser systems, which probe only the outermost electron levels. Furthermore, the short timescales associated with atomic motion (100s of fs) require much shorter pulses than the tens-to-hundreds of picoseconds provided by synchrotronbased x-ray sources. Finally, high brightness is needed to allow for single-shot diffraction or radiography to yield images at a discrete moment in time and study the progression of dynamic events. Development of a system capable of making measurements on these scales would open up regions of currently under-explored science, such as phase transitions in materials under shock loading and chemical reaction dynamics.

To this end, groups around the world have explored different mechanisms of x-ray production, such as ultrafast-laser-generated $\mathrm{K}_{\alpha}$ sources[1], $\mathrm{x}$-ray free-electron lasers (FELs) [2], electron bunch

slicing in synchrotrons[3], and relativistic Compton scattering[4]. While experimental measurements have been demonstrated using most of these techniques[5,6,7], the relative paucity of such highbrightness, ultrafast sources has limited their widespread application. There are also large regions of the energy spectrum where no bright source has been demonstrated.

The Picosecond Laser-Electron InterAction for the Dynamic Evaluation of Structures (PLEIADES) project was designed to provide ultrashort (100s of fs to a few ps), high brightness $\left(10^{19}\right.$ photons $\left./ \mathrm{mm}^{2} / \mathrm{s} / \mathrm{mrad}^{2} / 0.1 \% \mathrm{BW}\right) \mathrm{x}$-ray pulses with tunable photon energies from $10-100 \mathrm{keV}$ 
for single-shot diffraction and radiography experiments in high- $Z$ materials. To meet these design goals, in particular the tunability, we chose the relativistic Compton scattering mechanism.

The Compton scattering process (also commonly referred to as Thomson scattering since for most such sources, including PLEIADES, the scattering photon energy is well below the mass of the electron in its rest frame) was first studied in depth in the 1940s, when it was postulated as a stopping mechanism for intergalactic electrons.[8] It was not until the invention of the laser in the 1960s, however, that the first Compton sources were proposed[9] and demonstrated.[10] In a linear Compton scattering source, the x-rays are generated by scattering high-intensity laser light off a beam of relativistic electrons. In their rest frame, electrons are subjected to the electromagnetic of the laser that is Doppler upshifted, scattering the light in a classic dipole radiation pattern. Returning to the lab frame gives a second upshift, resulting in scattered light with a photon energy of

$$
h v_{\text {Scatt }}=2 \gamma^{2} \frac{1-\cos \varphi}{1+\gamma^{2} \theta^{2}} h v_{\text {Laser }},
$$

where $h$ is the Planck constant, $v_{\text {Scat }}$ and $v_{\text {Laser }}$ represent the peak scattered x-ray frequency and original laser frequency, respectively; $\varphi$ is the angle between the electron and the laser beams, $\theta$ is the x-ray observation angle relative to the electron trajectory, and $\gamma$ is the relativistic factor. The precise intensity and spectral profile of the resultant x-ray beam is closely linked to the laser frequency and bandwidth, the e-beam emittance, energy, and energy spread, and the interaction and focusing geometries. A more complete theory that takes all this into account is discussed by Hartemann et al.[11]

After the initial demonstrations, interest in producing Compton sources waned due to the low brightness of the $\mathrm{x}$-rays produced - a consequence of the low cross-section for Compton scattering $\left(\sigma_{\mathrm{T}}=7 \times 10^{-25} \mathrm{~cm}^{2}\right)$. In recent years, however, powerful lasers, photoinjectors, and high-quality 
accelerators have become commonplace, and the possibility of producing a high x-ray flux has renewed interest in the field. A variety of theoretical research into so-called "Laser Synchrotron Sources" was done[12], and in 1996 the first subpicosecond hard x-ray source was demonstrated at Lawrence Berkeley National Laboratory[4]. Additional Compton scattering results have been obtained by others.[13]

The PLEIADES facility requires the simultaneous integration of two laser systems and a new rf photoinjector with the existing 100-MeV Linear Accelerator at Lawrence Livermore National Laboratory (LLNL). The temporal overlap requirements necessary to produce Compton X-rays mean the systems have to have less than 2 ps of temporal jitter (for a $90^{\circ}$ interaction geometry) and less than $20 \mu \mathrm{m}$ of spatial jitter at the interaction point. With its current operating parameters, PLEIADES produces $\sim 1.6 \times 10^{6}$ photons/shot, which agrees with the theoretical prediction of $2.3 \mathrm{x}$ $10^{6}$ photons/shot for our current configuration. This level of x-ray flux per shot is comparable to the total number of x-rays used in other ultrafast diffraction measurements. For example, Ref. 5 discusses measurements of structural dynamics of InSb using X-ray diffraction. In that LBNL experiment, a source which produces $\sim 10^{4} \mathrm{x}$-rays was used, and the results averaged over $\sim 10^{4}$ shots. The PLEIADES source has improved on this flux by 2 orders of magnitude per shot. In a 3 x 3 $\operatorname{mrad}^{2}$ x $10 \mathrm{eV}$ phase space volume, PLEIADES currently produces $\sim 10^{3}$ photons, a bit over an order of magnitude from of the flux of $5 \times 10^{4}$ routinely used by K $\alpha$ sources for similar measurements [6]. Planned upgrades over the next year are expected to further improve the flux to $10^{8}$ photons/shot. The remainder of this paper consists of four sections. The first two describe the laser and electron systems, respectively, with particular emphasis on the unique requirements of a Compton source. The third section explains how the interaction region is designed and how overlap is achieved. The 
final section reviews the measured $\mathrm{x}$-ray results to date, compares those results with theoretical predictions, and discusses the upgrades planned to achieve our target x-ray brightness.

\section{LASER FACILITY}

Figure 1 shows the overall layout of the PLEIADES facility. The laser system used for these experiments, known as the FALCON laser, is a Ti:Sapphire chirped-pulse amplification (CPA) based system capable of producing over $1 \mathrm{~J}$ of uncompressed 820-nm light. The laser system begins with a Compact C20s Ti:Sapphire oscillator from Femtosource, which produces 30 fs pulses with a bandwidth of $36 \mathrm{~nm}$ centered at $815 \mathrm{~nm}$. This mirror-dispersion-controlled Kerr-lens mode-locked laser also serves as the master clock for the entire experimental facility.

A photodiode monitors the output pulse train of the oscillator, and that signal is compared to a reference $81.557 \mathrm{MHz}$ signal from a crystal oscillator in a Time-Bandwidth CLX-1000 timing stabilizer. This box controls a picomotor and piezo crystal attached to the end mirror of the oscillator cavity, and adjusts the cavity length to keep the frequency stable. The photodiode signal is also filtered to produce a sinusoidal waveform that is frequency multiplied in a phase-locked dielectric resonant oscillator to $2.8545 \mathrm{GHz}$, which is then used to seed the rf amplifier for the linear accelerator, ensuring phase-lock between the laser and electron systems.

The oscillator pulses are stretched to $680 \mathrm{ps}$ in an all-reflective parabolic-mirror based expander.[14] The pulse train is then split with a dielectric beam splitter into two beams, with $30 \%$ of the light being coupled into a fiber to seed the photoinjector laser, and the remaining $70 \%$ used to seed the FALCON laser. Because the same oscillator seeds both laser systems, minimal timing jitter between the systems is assured. 
In the FALCON laser, the oscillator pulses are amplified to an energy of $7.3 \mathrm{~mJ}$ in a standard linear regenerative amplifier cavity, pumped with $45 \mathrm{~mJ}$ of $532 \mathrm{~nm}$ light from a Spectra-Physics GCR-190 laser. Following the regenerative amplifier is a 4-pass amplifier, which is pumped with the remaining $212 \mathrm{~mJ}$ of light from the GCR-190. The infrared input into the amplifier is monitored by two cameras, imaging the near and far field spots of the beam. A closed loop control system adjusts the pointing and centering of the beam via stepper motors on two mirrors to maintain alignment into the amplifier. The output energy of this amplifier is $68 \mathrm{~mJ}$. This beam is then sent into a second 4pass amplifier, which also has a closed-loop feedback system to maintain input alignment. This amplifer is pumped by $2.3 \mathrm{~J}$ of 532-nm light from a SpectraPhysics QuantaRay PRO-350 laser as well as a $1 \mathrm{~J}, 532 \mathrm{~nm}$, Q-switched, Nd:YAG, pump laser manufactured by Continuum, and produces

\section{$1.2 \mathrm{~J}$ of uncompressed light.}

The amplified light is upcollimated to a $1 / \mathrm{e}^{2}$ radius of $42 \mathrm{~mm}$, and the beam is then relay imaged, using two telescopes, $52 \mathrm{~m}$ to a vacuum chamber near the accelerator system, where it is compressed in a double-pass grating-compressor. GRENOUILLE[15] measurements of the compressed pulses at low power give a pulse length of $54 \mathrm{fs}$ with a phase retrieval error of 0.006 . The compressed pulse then propagates $20 \mathrm{~m}$ to the final focusing optics. The loss through the transport and compressor is $45 \%$, leaving up to $540 \mathrm{~mJ}$ available in the interaction region.

\section{LINEAR ACCELERATOR}

\section{A. The Photoinjector Laser System}

The electron beam that is used for these experiments is produced at the LLNL 100-MeV linear accelerator (linac), which we have substantially upgraded to meet our emittance and timing jitter requirements. The most significant upgrade was the installation of a new photoinjector on the 
accelerator, as an alternative to the preexisting thermionic injector. In a photoinjector, the electron bunch is produced via the photoelectric effect when a uv laser pulse strikes the cathode, liberating electrons. Thus by seeding the laser that drives the photoinjector,(the photoinjector laser system, or PLS), with the same laser oscillator that seeds the FALCON laser, the injection time of the electrons into the linear accelerator can be synchronized with the FALCON laser pulse. A second major benefit of the photoinjector is that it allows for much higher current density electron beams. This gives a much smaller initial spot, and corresponding lower emittance, as well as a high ( 100 A) current. Additionally, the accelerating gradients in a photoinjector $(\sim 100 \mathrm{MeV} / \mathrm{m})$ are generally much greater than in thermionic guns. This rapid acceleration limits emittance growth due to space charge effects before the beam reaches relativistic velocities.

The PLS was installed as close as practical to the linear accelerator and seeded through a 50meter, single-mode fiber with $30 \%$ of that light being split off from the main oscillator pulse. After coupling and transport losses, the seed light has an average power of $7.3 \mathrm{~mW}$, or $90 \mathrm{pJ}$ per pulse, which is coupled into a linear regenerative amplifier cavity. The Ti:Sapphire crystal in this amplifier is pumped with $50 \mathrm{~mJ}$ of 532-nm light from a DCR-2 laser. The end mirror leakage of this amplifier is monitored with a fast photodiode, which provides the trigger timing for the streak camera systems discussed later. This system produces $5.9 \mathrm{~mJ}$ ir pulses at $10 \mathrm{~Hz}$. Following the regenerative amplifier is a bow-tie configuration 4-pass power amplifier, similar to the two discussed for the FALCON system. The Ti:Sapphire crystal is pumped with the $280 \mathrm{~mJ}$ of laser light from the DCR-pump that is not sent to the regen, and amplifies the regenerative amplifier output up to the $90 \mathrm{~mJ}$ level. Again, this amplifier has a pointing and centering system to align the regenerative amplifier light entering it. 
The light from the 4-pass is then sent into the compressor. The pulse is not fully compressed to its transform limit, but is instead left at a pulse length of approximately $10 \mathrm{ps.} \mathrm{Simulations} \mathrm{of} \mathrm{the}$ electron beam in the photoinjector show that the best quality beams (in terms of energy spread and emittance) occur when using laser pulses with pulse lengths of a few ps to produce electrons from the photocathode. This is a result of the Coulomb repulsion of the electrons, which are initially created at rest. As the laser pulse gets shorter, the electron density increases until the repulsive forces start overwhelming the accelerating forces and causes the beam to spread. This longer pulse also has the advantage of minimizing the effects on the laser pulse temporal structure resulting from the residual cubic phase distortions introduced by the $50-\mathrm{m}$ fiber that transports the laser oscillator pulse to the PLS.

Following compression, the pulse is frequency-doubled in a Type I $\beta$-barium borate (BBO) crystal, then tripled in a second Type I BBO crystal to $269 \mathrm{~nm}$. We use a special waveplate between the harmonic crystals that rotates the polarization of the second harmonic a half-wave to align it with the fundamental for sum-frequency mixing. Generally, about $1.2 \mathrm{~mJ}$ of uv light is available, however, to prevent damage to the cathode in the photoinjector, the system is often turned down to provide only about $500 \mu \mathrm{J}$ of light at the tripler output. This uv pulse is then clipped with an aperture to a diameter of $2 \mathrm{~mm}$ to provide a hard-edged uv spot, which improves the emittance of the photoinjector. The aperture plane is relay imaged $50 \mathrm{~m}$ to the photoinjector cathode. The measured uv pulse width is 7 ps.

\section{B. The Linear Accelerator}

The rf photoinjector used to produce the electron beam for PLEIADES is based on a 1.6-cell standing wave geometry[16]. A pulse of S-band (2.8545 GHz) rf input with 7-MW peak power and 
3- $\mu$ s pulse length produces a peak standing wave electric field of up to $100 \mathrm{MV} / \mathrm{m}$ that accelerates the electrons to $5 \mathrm{MeV}$ in a distance shorter than $10 \mathrm{~cm}$. Focusing solenoids are employed in the photoinjector to preserve the transverse emittance[17] of the electron beam immediately off the cathode and to help match the electron beam into the accelerating sections. Currently, the gun is being operated with a more conservative accelerating gradient of $80 \mathrm{MV} / \mathrm{m}$ to avoid any possible damage due to electrical arcing, which also leads to lower quantum efficiency on the photocathode.

The electron bunch charge is determined by the pulse parameters of the PLS laser and the quantum efficiency of the photocathode. The 266-nm laser pulse is imaged to a 1-2-mm spot on a copper photocathode near the rf field peak, and electrons are currently produced with a quantum efficiency of approximately $8 \times 10^{-6}$ electrons/photon, which yields an electron bunch charge between $250-350 \mathrm{pC}$.

The electron bunch length is a function of the laser pulse duration, bunch charge, and accelerating voltage, and is typically a few picoseconds long, although we have measured bunch lengths as short as 300-fs with reduced bunch charges and active bunch compression. Because the PLS, which is directly responsible for initiating the electron bunch, and FALCON are seeded by the same oscillator, the timing of the electron beam is synchronized to the laser pulse that is to be scattered.

The beam generated by the photoinjector is coupled into the pre-existing $100-\mathrm{MeV}$ linear accelerator[18], where it is accelerated to energies ranging between 40 and $100 \mathrm{MeV}$ in four of the five truncated Stanford Linear Acceleratior Center-type traveling wave accelerating sections in the system.

After passing through the interaction area, the electron beam is deflected by a $30^{\circ}$-bend dipole 
magnet. This dipole serves as a spectrometer, yielding a measurement of the electron energy and energy spread, and also separates the e-beam from the scattered x-rays, which propagate in the same direction as the electrons. Following the dipole, the electron beam is absorbed in a Cu electron dump that is calibrated and provides a measure of the electron bunch charge. The electron dump is housed in a 10-cm-thick lead enclosure to minimize the effect of bremsstrahlung on the diagnostics.

\section{IV.INTERACTION REGION}

\section{A. Interaction geometry}

In performing a Compton scattering interaction, there are two basic geometries: collinear (or anticollinear) laser and electron beams, or non-collinear beams. The main advantage of a noncollinear geometry is the very short x-ray pulse length that can be generated. The duration of the generated x-rays, to first order, is the transit time of the laser pulse through the electron bunch. In a collinear geometry with an ultrashort $(<100 \mathrm{fs})$ laser pulse, this is essentially the duration of the electron bunch, generally on the order of a few picoseconds. In a $90^{\circ}$ interaction, the duration becomes a convolution of the laser pulse length and the electron beam diameter, and is only on the order of a few hundred femtoseconds.

The main disadvantages of a $90^{\circ}$ interaction geometry are a) a lower x-ray flux, a result of the fact that the laser pulse only interacts with some of the electrons, and b) more stringent timing requirements [Fig. 2]. In a $180^{\circ}$ interaction geometry, with a 50-fs, $20-\mu \mathrm{m}$ diameter laser pulse and a 2-ps, 20- $\mu \mathrm{m}, 5$-mm-mrad emittance electron beam, the full-width at half-maximum (FWHM) of the $\mathrm{x}$-ray flux produced as a function of the delay between the arrival of the laser pulse and the arrival of the electron bunch at the focus is $15 \mathrm{ps}$. For the same beams in a $90^{\circ}$ geometry, the FWHM is only 2 ps, and the number of x-rays produced drops by a factor of 10. Even at a shallow interaction angle of 
$172^{\circ}$, the interaction window drops to $2.3 \mathrm{ps}$. The much larger interaction window in the $180^{\circ}$ geometry results from the fact that the Rayleigh range and beta function of the laser and electrons, respectively, are much longer than the actual bunch lengths. When the beams are collinear, they don't have to interact in the volume of space defined by the physical bunches, but only within the volume of space defined by the two focusing ranges. If one beam is delayed, the beams still collide, just further upstream or downstream of the main interaction point. Because of the numerous advantages of the $180^{\circ}$ interaction geometry, we chose to do our initial interactions in this configuration.

The layout of the PLEIADES interaction region is shown in Fig. 3 . The $400 \mathrm{~mJ}$ ir laser is focused off a $60^{\prime \prime}$ focal length, $12^{\circ}$ off-axis parabolic mirror. The focusing beam is then directed to the interaction region by a motor-controlled dielectric mirror, which allows for control of transverse alignment of the laser focus at the interaction point. The spot is observed to have a $1 / \mathrm{e}^{2}$ waist radius of $36 \mu \mathrm{m}\left(42.2 \mu \mathrm{m}\right.$ FWHM) and an $\mathrm{M}^{2}$ value of 1.64. The Rayleigh range, which defines the interaction region in a $180^{\circ}$ geometry, is $3.1 \mathrm{~mm}$. After interaction with the electrons, the laser beam propagates down the linac beamline and dumps its energy in the walls as it expands after the focus.

The electron beam is focused by a set of quadrupole magnets with a magnetic field gradient of up to $15 \mathrm{~T} / \mathrm{m}$. To aid alignment at the focus, two cross-oriented dipole magnets steer the beam into this magnetic lens. Because the off-axis parabolic mirror that focuses the laser is fixed, the longitudinal position of the interaction region is set by the laser focus, and we adjust the longitudinal position of the electron beam waist to the position of the laser focus using this electron focusing system. Measurements of the e-beam at the focus have shown a spot size of $50 \mu \mathrm{m} \mathrm{rms}$, and rms beam emittances $\varepsilon_{\mathrm{x}}=5 \mathrm{~mm}$ mrad and $\varepsilon_{\mathrm{y}}=13 \mathrm{~mm}$ mrad, which were measured using the standard 
quadrupole scan technique. The beta function of the electron beam is $\sim 1 \mathrm{~cm}$. The quadrupole focusing was designed to minimize the spot size given the quadrupole magnets we had. Because the length of both the laser and electron bunches were much smaller than the Rayleigh range and beta functions, respectively, these parameters did not impact the design.

\section{B. Aligment}

Spatial alignment of the two focal spots was performed with the aid of an optically polished 0.3 " aluminum cube. The cube is mounted on a three-axis translation stage with its faces oriented vertically normal to the beamline, and horizontally at $45^{\circ}$ to the beamline. Because the laser beam reflects from the surface, the focus at the surface of the cube can be imaged into a charge-coupled device (CCD) camera. To avoid damaging the cube or camera, the ir laser pulse energy is reduced by a combination of turning off the pump lasers to the two 4-pass amplifiers and inserting neutral density filters to attenuate the beam by a factor of $10^{8}$. When the electron beam strikes the cube, it produces optical transition radiation (OTR), which can also be imaged by the CCD camera. The vertical alignment of the two beams is then readily apparent, and the horizontal alignment is determined by positioning the cube such that both beams just hit the cube edge. Generally, our procedure is to place the cube at the laser focus, optimize the electron beam focus on the cube, and steer the laser beam laterally to align to the optimal electron beam position.

Temporal alignment is more complex than the spatial alignment because the propagation times for the FALCON laser and the PLS/electrons, which are set by path lengths that are approximately 70 meters long, must be matched to within a few picoseconds. There are three steps to the initial synchronization. First, a beam-current pickoff and an ir photodiode are used to determine the initial timing. The electron beam propagating through the interaction area generates a short pulse magnetic 
field, which produces current in the two 100-ohm junctions of the pickoff. The generated signal is then detected by an oscilloscope as $\sim 150$ ps FWHM pulses. Similar accuracy is obtained for the arrival time of the laser by using a fast infrared UHS 016 photodiode. By selecting a different oscillator pulse to switch into the FALCON regenerative amplifier, we are able to get the electron and laser arrival time difference to less than $12 \mathrm{~ns}$ (the spacing between pairs of oscillator pulses).

Second, for more accurate timing, we use a Nikon Nikkor $50 \mathrm{~mm}$ f/1.4 lens to image the OTR and the laser light reflected from the cube onto the $100-\mu \mathrm{m}$ slit on an Imacon 500 Series streak camera. This camera uses an S20 photo-cathode with a quantum efficiency greater than 5\% over the visible wavelengths, which makes simultaneous streaking of the OTR and drive laser light possible. Using a combination of this streak camera and the current pick-off/photodiode signals, the laser and electron paths are brought to within a few tens of picoseconds by manually sliding the retroreflecting roof mirror in the FALCON compressor along a 2-m rail. Because this mirror is located between the second and third grating strike in the compressor, its position does not have a significant effect on the compressed pulse.

The third and final stage of temporal alignment is performed using the streak camera at its highest sweep speed, $18.7 \mathrm{ps} / \mathrm{mm}$, which gives us temporal resolution of 2-5 ps, limited by the spacing on the microchannel plate that is used as an amplifier for the output phosphor and by the entrance slit size. Using this signal and a motorized stage under the same roof mirror in the compressor, we can synchronize the laser and electron beam arrival to the resolution of the streak camera. This measurement also gives us the relative timing jitter, which is seen to be below the streak camera resolution. Attempts at further optimizing the timing by maximizing the x-ray signal directly as a function of the delay between the pulses yield no improvement, indicating that the 
temporal overlap achieved with the 2-ps resolution of the streak camera was sufficient for the $180^{\circ}$ interaction geometry.

\section{X-RAY OBSERVATION AND THEORETICAL COMPARISON}

A variety of diagnostics are available for detection of the Compton x-rays. An x-ray CCD is the primary diagnostic, and consists of a $145-\mu \mathrm{m}$ thick $\mathrm{CsI}(\mathrm{Tl})$ scintillator that is coupled by a fiber optic bundle to an optical Princeton Instruments 16-bit, 1340x1300-pixel CCD chip, with a demagnification of $3: 1$. The chip size is $2.54 \times 2.54 \mathrm{~cm}$, which gives a field of view as large as $7 \times 7 \mathrm{~cm}$. The scintillator, which is protected by a $0.5-\mathrm{mm}$ Be filter, provides a photon detection quantum efficiency of 0.4 at $60 \mathrm{keV}$. We calibrated the x-ray CCD using a 59.5-keV Am 241 radioactive source. We calibrated the source itself using a single-photon counting $\mathrm{Ge}(\mathrm{Li})$ detector with a quantum efficiency for the energies of interest that is close to $100 \%$. To obtain an accurate wavelength measurement of the x-rays, we also plan on fielding an AmpTek XR-100CR x-ray diode, with a $25 \mathrm{~mm}^{2}$ active area, and a bent-crystal x-ray spectrometer [19].

The total number of x-rays produced is calculated from the x-ray CCD image (Fig. 4a) and the theoretically predicted spectrum. The observed beam profile is fit with a 2D-Gaussian (Fig. 4b), and that Gaussian fit is integrated to give the total number of observed photons from the scintillator. The number of photons observed correlates directly to the x-ray energy deposited in the scintillator $\left(E_{d e p}\right)$ through the CCD calibration of 0.12 photons per $\mathrm{keV}$ deposited. To convert the measured energy into an estimate of the number of x-rays produced, the $\mathrm{x}$-ray losses have to be accounted for. The total theoretical spectrum, $N_{x}\left(\omega_{s}\right)$, is multiplied by the energy-dependent transmission through the 0.5 " thick fused silica mirror used to align the laser to the interaction point $\left(T_{F S}\left(\omega_{s}\right)\right)$, and by the energy-dependant probability of interaction in the $\mathrm{CsI}(\mathrm{Tl})$ scintillator $\left(\eta_{C s I}\left(\omega_{s}\right)\right)$. The total energy 
deposited in the CsI crystal is then

$$
E_{t o t}=\int \eta_{C s I}\left(\omega_{s}\right) T_{F S}\left(\omega_{s}\right) N_{X}\left(\omega_{s}\right) \mathrm{h} \omega_{s} d \omega_{s}
$$

and so the total number of X-rays produced, as a function of energy deposited in the CsI, is

$$
N_{X}=\frac{\int N_{X}\left(\omega_{s}\right) d \omega_{s}}{E_{t o t}} E_{d e p}
$$

For initial x-ray production, the FALCON laser was pumped with only one of the two pump lasers, resulting in only $400 \mathrm{~mJ}$ of uncompressed light, or $180 \mathrm{~mJ}$ at the interaction point. The electron beam was run at various energies between 54 and $57 \mathrm{MeV}$, corresponding to expected peak $\mathrm{x}$-ray energies of $70-78 \mathrm{keV}$, with $\sim 270 \mathrm{pC}$ of charge in each electron bunch. Early runs were made with $40 \mathrm{~mJ}$ of ir energy scattering off the electron beam, due to a misalignment of the grating compressor that resulted in a line focus at the interaction point. In these runs, a total x-ray flux of $5 \times 10^{4}$ photons/shot was generated. Subsequent improvements to the laser spot yielded an X-ray flux of $1.3 \times 10^{6}$ photons/shot, compared with a theoretical value of $2.3 \times 10^{6}$.

The measured rms divergence of the $\mathrm{x}$-ray beam is $3.0 \mathrm{mrad}$ in the $\mathrm{x}$ dimension, and $4.7 \mathrm{mrad}$ in the $y$ dimension. The difference in the x-ray divergences for the two dimensions arises from a combination of the asymmetric emittance in the electron beams and the polarization direction of the laser. Figure 5 shows the theoretical x-ray profile compared with the measured profile, which shows good agreement. The theoretical profile is based on simulations for the electron and laser parameters measured during the run, quoted above, and corrected for the fused silica transmission and CsI response, as discussed above. The cause of the discrepancy is unclear, but is most likely due to inaccuracies in determining the emittance of the interacting electrons, which was used for the theoretical calculation. The e-beam emittance has a significant impact on the x-ray divergence. 
As a demonstration of the strong angular dependence of the spectrum, a 0.005 " tantalum foil was placed in the x-ray path, in front of the x-ray CCD. Tantalum has a k-edge at $67.46 \mathrm{keV}$, and so strongly attenuates x-rays just above that energy. Due to the correlation between divergence angle and energy, this corresponds to attenuation of the central part of the beam. Figures $6 \mathrm{a}$ and $6 \mathrm{~b}$ show the theoretical beam profiles at two different e-beam energies, 55 and $57 \mathrm{MeV}$, which correspond to 73.1 and $78.5 \mathrm{keV} x$-ray peak energies, respectively. Figures $6 \mathrm{c}$ and $6 \mathrm{~d}$ then show the actual recorded profiles for similar conditions, and show good qualitative agreement with the predictions. This similarity is a strong indicator that the spectrum is close to what we expect from a Compton source.

\section{VI.CONCLUSIONS}

To date, we have demonstrated successful Compton scattering in the $180^{\circ}$ geometry using the PLEIADES system at LLNL, and observed x-rays fluxes and beam profiles that agree well with the theoretically predicted values. The next step is measuring the total spectrum, as well as the spectrum as a function of angle, which will allow us to establish our spectral brightness. This will be done with the XR-100CR diode, as well as the bent-crystal spectrometer.

The x-ray flux needs to be increased by 2 orders of magnitude to reach the design goal. A factor of 3 will come from increasing the FALCON output laser to full design power, giving $540 \mathrm{~mJ}$ at the interaction point instead of $180 \mathrm{~mJ}$. Also, the e-beam bunch charge can be increased by turning up the uv pulse energy, which we are currently running below our design parameters to minimize the risk of damaging the photocathode while initial results are gathered. The most significant improvement will come from the installation of new permanent magnet quadrupoles for the final electron beam focus. This should allow us to produce a $15 \mu \mathrm{m}$ rms spot size at the focus, which will 
significantly improve the laser/electron beam overlap, allowing us to achieve the desired goal of $10^{8}$ photons/shot.

\section{ACKNOWLEDMENTS}

This work was performed under the auspices of the U.S. Department of Energy by University of California, Lawrence Livermore National Laboratory under Contract W-7405-Eng-48. UCRL-JP200521. 


\section{REFERENCES}

1 T. Guo, Ch. Spielmann, B. C. Walker, C. P. J. Barty, Rev. Sci. Inst. 72, 41 (2001).

2 J. Arthur, G. Materlik, R. Tatchyn, H. Winick, Rev. Sci. Inst. 66, 1987 (1995).

3 R. W. Schoenlein, S. Chattopadhyay, H. H. W. Chong et al., Science 287, 2237 (2000);

A. A. Zholents and M. S. Zolotorev, Phys. Rev. Lett. 76, 912 (1996).

4 R. W. Schoenlein, W. P. Leemans, A. H. Chin et al., Science 274, 236 (1996).

5 A. H. Chin, R. W. Schoenlein, T. E. Glover, P. Balling, W. P. Leemans, C. V. Shank, Phys. Rev. Lett. 83, 336 (1999).

6 C. Rischel, A. Rousse, I. Uschmann, et al., Nature 390, 490 (1997); A. Rousse, C.

Rischel, S. Fourmaux, et al., Nature 410, 65 (2001).

7 C. W. Siders, A. Cavalleri, K. Sokolowski-Tinten et al., Science 286, 1340 (1999); A.

Cavalleri, C. W. Siders, F. L. H. Brown et al., Phys. Rev. Lett. 85, 586 (2000).

$8 \quad$ E. Feenberg and H. Primakoff, Phys. Rev. 73, 449 (1948).

9 R. H. Milburn, Phys. Rev. Lett. 10, 75 (1963); F. R. Arutyunian, V. A. Tumanian, Phys. Lett. 4, 176 (1963).

10 G. Fiocco, E. Thompson, Phys. Rev. Lett. 10, 89 (1963); C. Bemporad, R. H. Milburn, N. Tanaka, M. Fotino, Phys. Rev. 138, B1546 (1965).

11 F. V. Hartemann, H. A. Baldis, A. K. Kerman, A. Le Foll, N. C. Luhmann, Jr., B. Rupp, Phys. Rev. E 64, 016501 (2001); F. V. Hartemann, High Field Electrodynamics (CRC Press, Boca Raton, FL, 2002).

12 P. Sprangle, A. Ting, E. Esarey, A. Fisher, J. Appl. Phys. 72, 5032 (1992); I. V. Pogorelsky, Nuc. Inst. Meth. Phys. Res. A, 411, 172 (1998); E. Esarey, S. K. Ride, P. 
Sprangle, Phys. Rev. E 48, 3003 (1993); K. J. Kim, S. Chattopadhyay, C. V. Shank, Nuc. Inst. Meth. Phys. Res. A 341, 351 (1994); S. K. Ride, E. Esarey, M. Baine, Phys. Rev. E 52, 5425 (1995).

13 I. V. Pogorelsky, T. Ben-Zvi, T. Hirose et al., Phys. Rev. Spec. Topics-Accel. \& Beams 3 090702 (2000); M. Yorozu, J. F. Yang, Y. Okada, T. Yanagida, F. Sakai, A. Endo, Japanese J. of Appl. Phys. Part 1 40, 4228 (2001); T. Nakano, J. K. Ahn, M. Fujiwara et al., Nuc. Physi. A 684, 71C (2001); A. Ting, R. Fischer, A. Fisher et al., J. Appl. Phys. 78, 575 (1995).

14 P. S. Banks, M. D. Perry, V. Yanovsky, S. N. Fochs, B. C. Stuart, J. Zweiback, IEEE J. Quant. Elect. 36, 268 (2000).

15 P. O'Shea, M. Kimmel, X. Gu, R. Trebino, Opt. Lett. 26, 932 (2001); P. O'Shea, M. Kimmel, R. Trebino, J. Opt. B 4, 44 (2002).

16 G. P. Le Sage, S. G. Anderson, T. E. Cowan, J. K. Crane, T. Dimire, J. B. Rosenzweig, Proceedings of The Ninth Workshop on Advanced Accelerator Concepts, Sante Fe, New Mexico, 2000, edited by P. L. Colestock, S. Kelley (AIP, 2001), Vol. 569, p. 391.

17 B. E. Carlsten, Nuc. Inst. Meth. Phys. Res. A 285, 313 (1989).

18 S. C. Fultz, C. L. Whitten, IEEE Trans. Nuc. Sci. 18, 533 (1971).

19 L. T. Hudson, A. Hennis, R. D. Deslattes et al., Rev. Sci. Inst. 73, 2207 (2002). 


\section{IX.FIGURE CAPTIONS}

Figure 1 - An overall schematic of the PLEIADES facility. The oscillator signal serves as a source for the accelerator rf, as well as seeding both the FALCON and PLS laser systems, to ensure synchronicity between the laser and electron beams in the interaction region.

Figure 2 - X-ray flux as a function of the difference between the laser and electron arrival times at the interaction region for (a) a $90^{\circ}$ interaction geometry and (b) a $180^{\circ}$ interaction geometry for the same beam parameters. This shows the lower flux and more stringent jitter requirements that result from a $90^{\circ}$ interaction.

Figure 3 - Diagram of the PLEIADES interaction region, showing the laser, electron, and x-ray beam paths. The x-rays, produced in the scattering of the laser off the electron beam, pass through the laser mirror as they exit the interaction region.

Figure 4 - [COLOR] (a) CCD image of the Compton x-ray beam. (b) Major and minor axis lineout of CCD image with 2D Gaussian fit shown. The rms divergence along the major axis is 6 mrad, and along the minor axis is 4 mrad.

Figure 5 - Comparison of the measured and theoretical x-ray profiles at the CCD camera. The transmission to the camera and the response of the scintillator are accounted for. The line is the theoretical profile, based on the measured laser and electron beam parameters and the transmission and response of the CCD camera, and the diamonds are the measured profile. Both the major and minor axes of the elliptical beam spot are shown.

Figure 6 - [COLOR] X-ray profile after transmission through 0.005" Tantalum foil. (a) Predicted profile with $55 \mathrm{MeV}$ e-beam energy. (b) Predicted profile with $57 \mathrm{MeV}$ e-beam energy. (c) Measured profile with $55 \mathrm{MeV}$ e-beam energy. (d) Measured profile with $57 \mathrm{MeV}$ e-beam energy. 


\section{FIGURES}

Figure 1

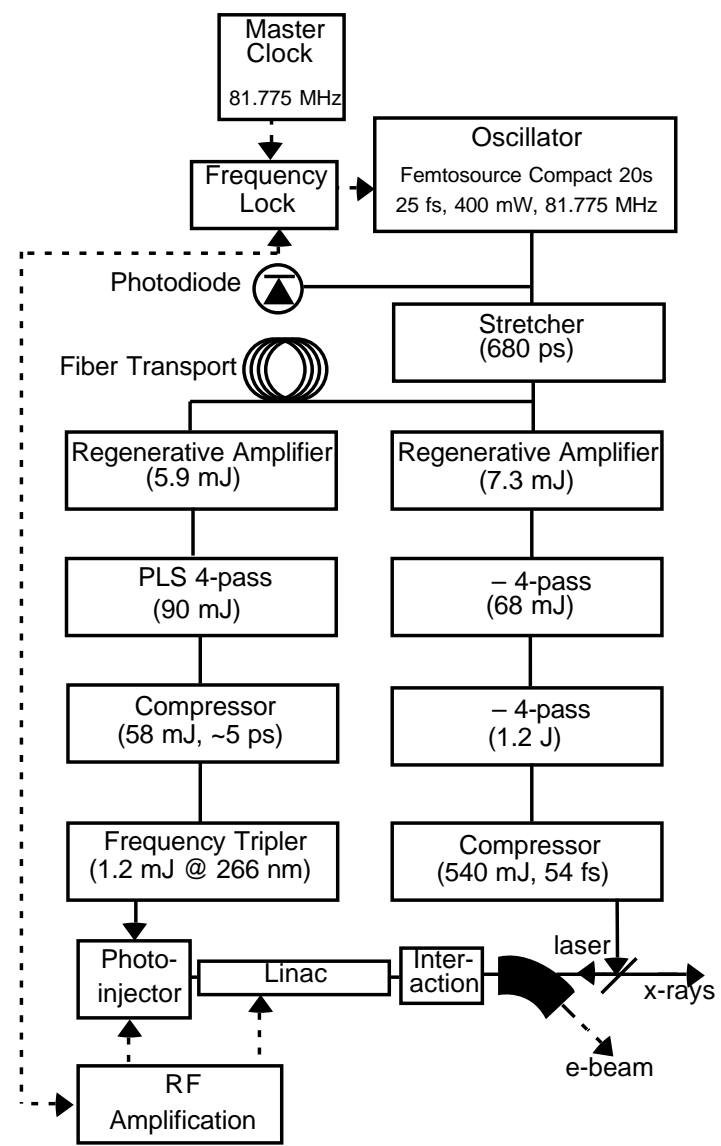


Figure 2

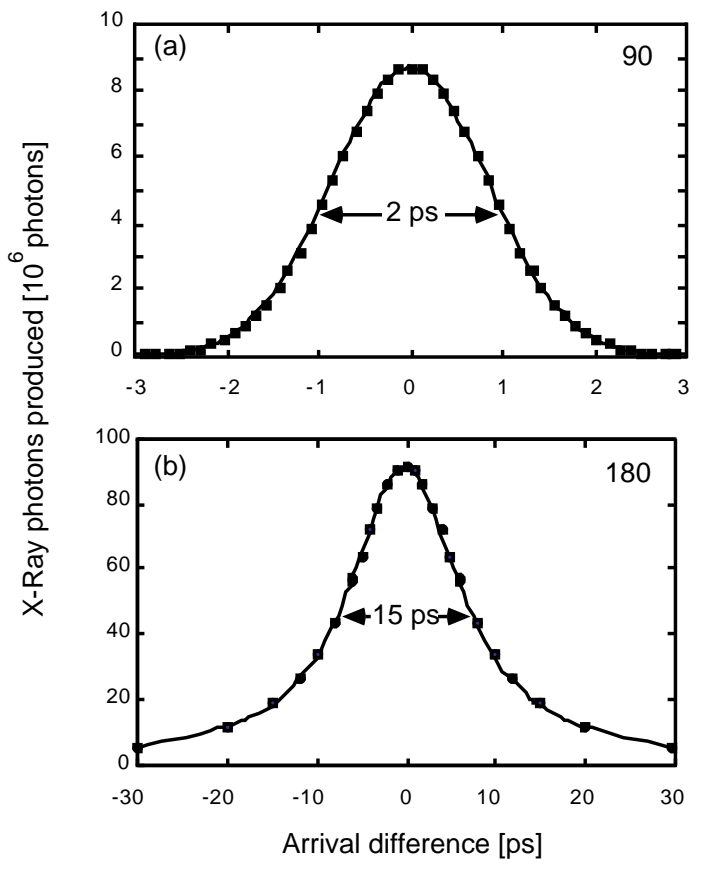


Figure 3

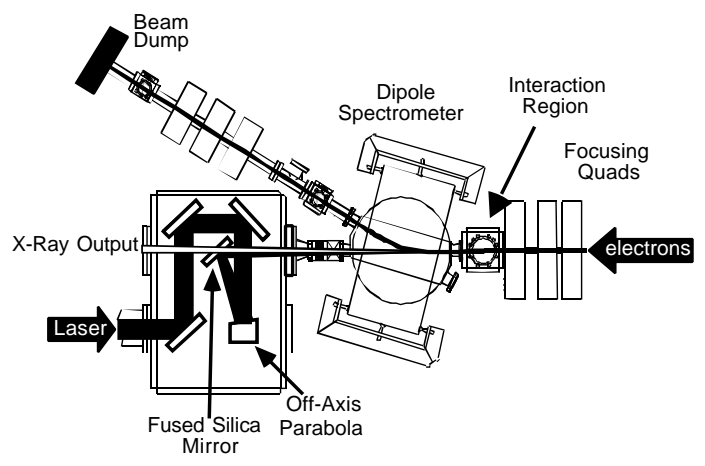


Figure 4
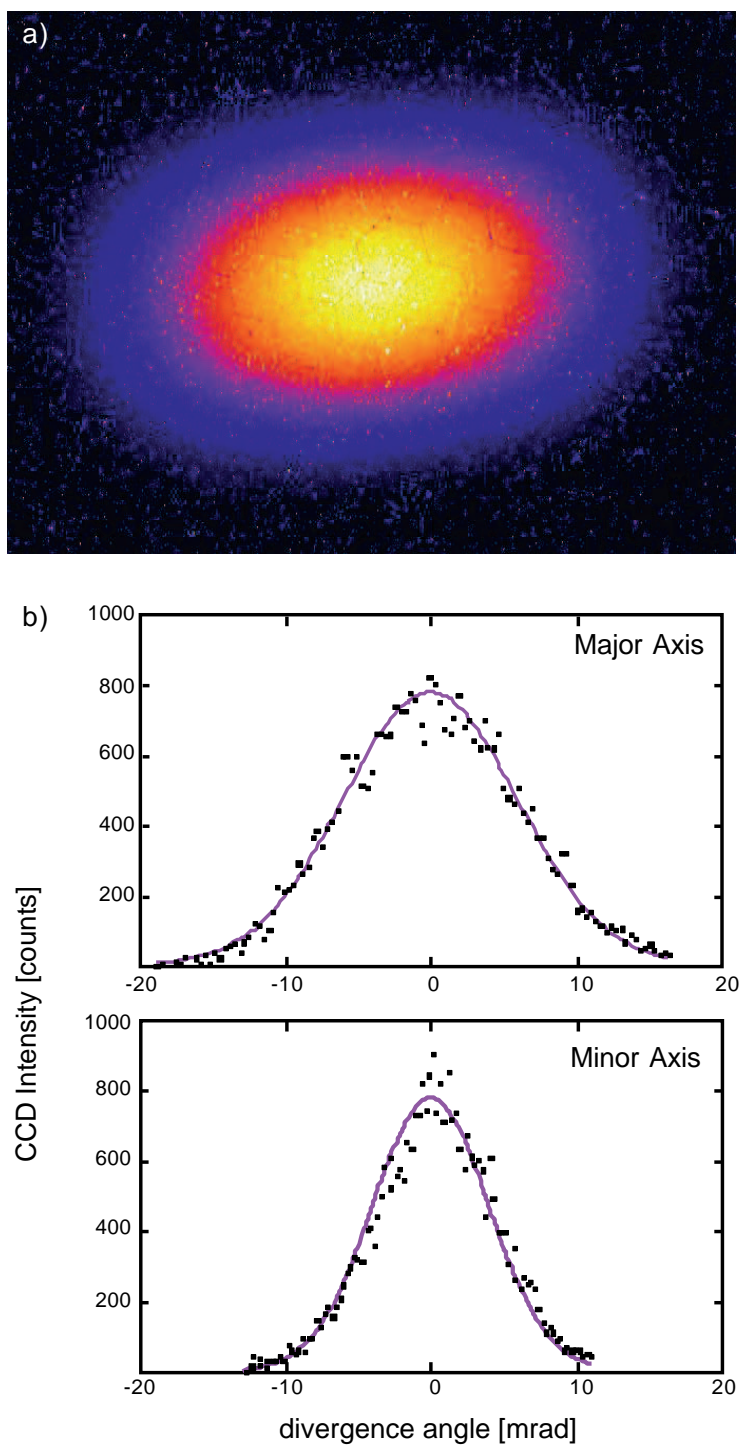
Figure 5

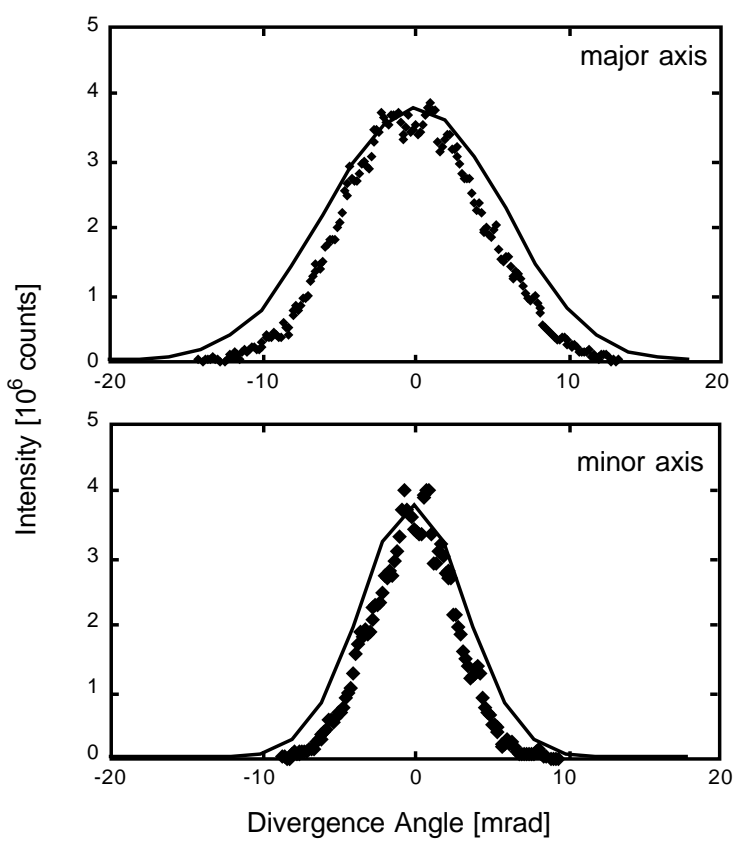


Figure 6

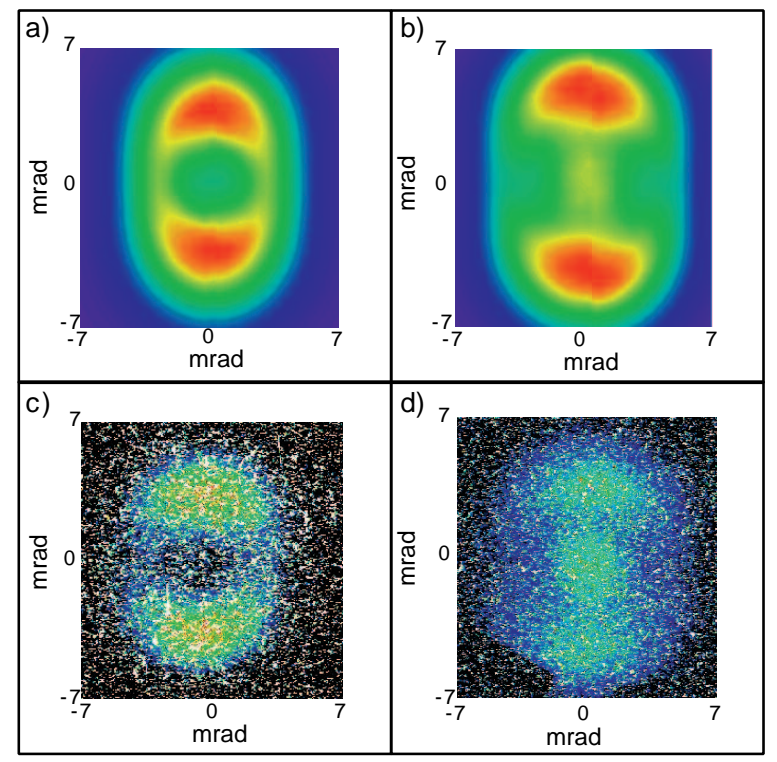

